\title{
Cent ans d'études balzaciennes au Japon
}

\section{Takao Kashiwagi}

\section{(2) OpenEdition}

\section{Journals}

\section{Édition électronique}

URL : http://journals.openedition.org/studifrancesi/35572

DOI : 10.4000/studifrancesi.35572

ISSN : 2427-5856

\section{Éditeur}

Rosenberg \& Sellier

\section{Édition imprimée}

Date de publication : 1 juillet 2005

Pagination : 65-72

ISSN : 0039-2944

\section{Référence électronique}

Takao Kashiwagi, « Cent ans d'études balzaciennes au Japon », Studi Francesi [En ligne], 145 (XLIX | I) | 2005, mis en ligne le 30 novembre 2015, consulté le 19 avril 2021. URL : http://

journals.openedition.org/studifrancesi/35572 ; DOI : https://doi.org/10.4000/studifrancesi.35572

\section{(c) (i) (2)}

Studi Francesi è distribuita con Licenza Creative Commons Attribuzione - Non commerciale - Non opere derivate 4.0 Internazionale. 


\section{Cent ans d'études balzaciennes au Japon'}

\section{La traduction japonaise de Balzac et les études balzaciennes au Japon.}

Avant d'aborder les études balzaciennes au Japon, commençons d'abord par faire l'historique de l'influence européenne sur la culture japonaise. Le Japon a ouvert ses portes aux étrangers en l'occurrence les Européens, vers 1855. La civilisation européenne a été assimilée très rapidement par la société japonaise. Le nouveau gouvernement, qui venait de triompher de la guerre civile, s'est efforcé pendant dix années d'introduire la civilisation et la culture européennes, d'abord celles de la France à laquelle s'était rallié l'ancien gouvernement du Shogunat des Tokugawa, et ensuite celles de l'Angleterre à laquelle le nouveau gouvernement de Satsuma et Tyoshu fut très redevable pour vaincre le Shogunat des Tokugawa.

Une connaissance profonde des lettres européennes était donc l'outil indispensable pour gravir l'échelle sociale. Voilà la raison de tant de traductions de livres européens. Le Tour du monde en quatre-vingt jours de Jules Verne a l'honneur d'être le premier roman français traduit en japonais. Songez au fait que cette traduction est apparue seulement quatre ans après la publication de l'original. Les intellectuels japonais avaient soif de lire les livres européens, d'une part pour acquérir des connaissances sur les coutumes et les institutions des pays occidentaux, d'autre part pour satisfaire leurs goûts littéraires qui n'avaient pas été satisfaits par la littérature japonaise à l'époque du Shogunat des Tokugawa, car il s'agissait d'une littérature moraliste qui voyait finalement triompher la vertu lors du dénouement du roman.

Des années 1890 jusqu'à 1900, c'était Zola et Maupassant qui avaient un prestige certain parmi les intellectuels, influencés bien sûr par la vogue mondiale de cette époque. La première traduction de Balzac est apparue à ce moment-là. C'est cette traduction d'Un épisode sous la Terreur par Kotyo Baba en 1897 qui a fait connaître le nom de Balzac romancier aux lecteurs japonais². La plupart de ses œuvres ont été traduites en japonais. Plus de quatre éditions des œuvres complètes de Balzac ont été publiées et une nouvelle édition de treize romans vient d'achever su publication. Nous pouvons compter 500 titres environ des romans de Balzac publiés par une soixantaine de traducteurs japonais. Il n'y a que quatre œuvres dans la Comédie humaine qui ne sont pas encore traduites en japonais: Le Député d'Arcis, L'Enfant maudit, Gaudissart II, Mầtre Cornélius. Balzac est certainement l'un des romanciers français le plus lu par les Japonais.

Quant aux études balzaciennes au Japon, nous pouvons citer La littérature française moderne écrite en 1914 par Suéo Goto comme le premier essai qui traite de Balzac au Japon. Après lui se succèdent jusqu'à aujourd'hui d'innombrables livres et articles écrits sur Balzac et ses œuvres, mais ils sont écrits en japonais ce qui les rend difficiles d'accès pour les lecteurs étrangers. Parmi eux, nous nous contentons de citer quelques livres importants: La Méthode de Balzac par Kiyotaro Wakazono (1941); Le Monde de Balzac par Hidéki Sugiyama (1942), Masao Yasushi, Etudes balzacienne

(1) Cet article a été d'abord écrit pour «Gallia» $\mathrm{XL}$, Société de langue et littérature française de l'Université d'Osaka, 2001. Il a été remanié et augmenté dans «Equinoxe» No. 19, Rinsen-Books, 2001.

(2) M. Hara cite une autre traduction d'Une
Passion dans le désert publiée trois ans auparavant dans sa Bibliographie des ceuvres de Balzac traduites en japonais et des critiques sur Balzac écrits en japonais, p. 30, Sourougadaï-Shuppansha, 1969. Mais on ne peut pas être sûr que ce texte est vraiment de Balzac, comme le titre en japonais est «le Tigre». 
- création de la Comédie humaine - (1960), Toru Terada, Balzac - du parterre de la Comédie humaine - (1967) et Les vieilles filles dans la Comédie humaine (1984), Kazuo Kiryu, Balzac-entre un génie et un philistin - (1975), Teruo Michimune, Exorde aux études des ouvres de jeunesse de Balzac tome I (1982) et tome II (1989), Mamoru Kaneko, La vie de Balzac (1987), Yasuhisa Yoshikawa, Balzac, romancier en lutte (1999), Séiichi Ishii, Le monde de Balzac (1999), Tetsuo Takayama, Balzac (1999), Takao Kashiwagi, Déchiffrer la Comédie humaine (2000). Michimune publie très récemment Etudes sur «la Comédie bumaine», tome I (2001).

Balzac - du parterre de la Comédie humaine - de Terada, composé en grande partie de ses introductions aux romans balzaciens qu'il a traduits, a exercé une grande influenœ sur les jeunes balzaciens japonais par son style original et par une analyse aussi fme qu'audacieuse. Terada, grand lecteur de Valéry et de Dostoïevski, y traite des œuvres de Balzac, telles que la Femme de trente ans, Mémoires de deujeunes mariées, Les Employés, César Birotteau, Les Parents pauvres, Un Début dans la vie, Les Paysans, etc. Il les considère non comme de simples fictions mais comme des problèmes réels et actuels posés par le romancier et il note les impressions et les opinions littéraires que lui suggère sa lecture. Cette attitude évoque celle d'Alain.

Le titre du livre de Michimune, Exorde aux études des ceuvres de jeunesse de Bal$z a c$, relève certainement de la modestie japonaise. Ce n'est pas un «exorde» à vrai dire, c'est une grosse étude qui compte presque mille pages en deux tomes. Michimune lit très soigneusement toutes les œuvres de jeunesse de Balzac depuis les Euvres de l'Abbé Savonati jusqu'aux épisodes de son Histoire pittoresque de la France et il y trouve les éléments précurseurs des chefs-d'œuvre de la Comédie bumaine. Les livres de Kiryu, Kaneko et Takayama traitent tous de la biographie de Balzac. Kiryu détaille surtout la formation du grand écrivain en citant adroitement ses lettres et ses œuvres. Kaneko retrace sérieusement la vie de Balzac en consultant plusieurs études biographiques françaises ainsi que les écrits du romancier et de ses contemporains.

L'an 1999 est vraiment l'année balzacienne au Japon; la publication des Chefsd'œuvre de Balzac en 13 volumes commence par Le Père Goriot traduit par Shigeru Kashima, et trois livres sur Balzac sont apparus presque en même temps que la commémoration du bicentenaire du romancier. Yasuhisa Yoshikawa le dépeint dans son Balzac, romancier en lutte comme un précurseur qui combat pour le droit des écrivains contre le capitalisme de l'époque. Il met également en relief chez Balzac un romancier qui adopte énergiquement les nouvelles tendances des sciences au début du dix-neuvième siècle, selon l'épistémè du dix-huitième siècle. Le monde de Balzac de Séiichi Ishii est une bonne introduction au monde littéraire de Balzac. Ishii y raconte les charmes des romans balzaciens et de Balzac en personne devant ses étudiants qui l'entourent, comme un grand maître livre le secret de son art à ses disciples. Tetsuo Takayama, qui a commencé ses études sur Balzac vers 1960 avec Kiryu et Ishii, a achevé son Balzac, biographie très documentée et écrite dans un style concis. Un an après, comme pour le cent-cinquantenaire de la mort du romancier, Takao Kashiwagi publie Déchiffrer la Comédie humaine, dans lequel il analyse une quinzaine d'œuvres de la Comédie bumaine, Facino Cane, Le Lys dans la vallée, Les Parents pauvres, Eugénie Grandet, etc. et tente d'éclaircir la structure de chaque récit merveilleusement conçu par le romancier. Etudes sur «la Comedie bumaine » de Muchimune est la suite de ses travaux sur les œuvres de jeunesse de Balzac. Michimune analyse presque chronologiquement chaque œuvre de la Comédie bumaine. Le tome I traite jusqu'au bal de Sceaux. On s'attend beaucoup à son accomplissement.

Nous ne pouvons pas passer aux études balzaciennes écrites en français par les Japonais sans faire mention du livre du bicentenaire de la naissance de Balzac publié en 1999 au Japon. Ce livre, intitulé Balzac, a été réalisé grâce à la bonne volonté des balzaciens japonais. Trente-quatre balzaciens japonais y ont consacré des articles et 
d'autres balzaciens ont partagé avec eux la plupart des frais de publication. Ajoutons qu'e est entrain d'éditer la version française de ce livre a été faite en 2000.

\section{Premiers travaux par les balzaciens japonais en France}

C'est Tetsuo Takayama qui a été le premier au Japon à publier ses études en français, Les Euvres romanesques avortées de Balzac (1829-1842). Takayamales a écrites d'abord comme thèse de Doctorat de l'Université de Paris sous la direction de Pierre-Georges Castex. Il l'a soutenue en 1962 à la Sorbonne. Rentré au Japon, il l'a remaniée pour perfectionner son travail et Keio Institute of Cultural and Linguistic Studies l'a publiée dans la collection Studies in the bumanities and social relations en 1966. Comme l'auteur le dit dans son Avant-propos, le but de ses études est «1 d'étudier chacune des œuvres avortées de Balzac en elle-même, $2^{\circ}$ de dégager les rapports de celles-ci avec les œuvres achevées, $3^{\circ}$ de mettre ainsi en lumière, dans la mesure du possible, la méthode de travail de Balzac, ainsi que les difficultés qu'il a rencontrées dans sa création littéraire» ${ }^{3}$.

Dans ce but, Takayama explore les manuscrits laissés par Balzac et il y poursuit les traces des idées de l'écrivain. Après avoir examiné minutieusement les projets de romans historiques, les œuvres avortées qui devaient être incluses dans les Contes et Romans philosophiques, c'est-à dire dans la Comédie bumaine, Takayama précise les étapes historiques de la rédaction du romancier et y remarque une tendance propre à la création littéraîre balzacienne selon laquelle «Balzac avait à élargir la dimension et à modifier le caractère d'un ouvrage au cours de l'exécution» ${ }^{4}$. Il suppose que la cause de l'échec dans l'accomplissement de ces œuvres tient à «l'étendue trop vaste du projet, la trop grande beauté du sujet» ${ }^{5}$. Cependant «la réussite brillante, l'accomplissement gigantesque de La Comédie bumaine n'a été possible qu'à la suite d'échecs successifs essuyés par Balzac».

Le livre de Takayama «n'est pas tant l'histoire des échecs littéraires de Balzac, comme le suggère son titre, que celle de la genèse souterraine de La Comédie bumaine» ${ }^{7}$. En fait, c'est un des ouvrages indispensables pour ceux qui veulent connâ̂tre le processus de l'élaboration des romans balzaciens. Il fournit jusqu'à nos jours des documents très précieux et nous enseigne l'importance des œuvres avortées du romancier, d'autant plus qu'on se lance aujourd'hui dans les études génétiques de La Comédie bumaine.

Trois ans après la parution du livre de Takayama, Yuko Nishikawa a soutenu sa thèse de doctorat de l'université de Paris sous la direction de Pierre-Georges Castex. Elle l'a remaniée et l'a publiée sous le titre Balzac et le Dandysme en 1977. Ce livre comporte deux parties: la première partie intitulée «Balzac en 1830, journaliste de mode et écrivain de mœurs» traîte des pensées balzaciennes sur la mode et sur le dandysme, en détaillant son article Traité de la vie élégante paru dans La Mode et la deuxième partie «La création romanesque des personnages de dandys dans La Comédie humaine» est consacrée aux principaux dandys balzaciens, Maxime de Trailles, Eugène de Rastignac, Charles Grandet, Don Juan Belbidéro, et entre autres Henri de Marsày.

Depuis Balzac et le mal du siècle de Pierre Barbéris (1970), les études balzaciennes s'orientent vers les rapports intimes entre la création littéraire du romancier et la

(3) T. Takayama, Les ceuvres romanesques avortées de Balzac (1829-1842), pp. VII-VIII, Keio Institute of Cultural and Linguistic Studies, 1966.

(4) Ibid., p. 129.
(5) Ibid., p. 131.

(6) Ibid., p. 133.

(7) S. Ishii, «Revue critique» in L'année balzacienne 1968, p. 459. 
culture de l'époque, qui l'entoure. Mais le livre de Nishikawa fait d'elle une pionnière dans ce domaine qui s'attache à l'activité journalistique de Balzac. Nishikawa parcourt les numéros de La Mode et des autres revues de mode de l'époque et éclaircit le statut du journalisme à l'époque de Balzac. Après avoir examiné en détail les personnes qui participent à la publication de La Mode, Emile de Girardin, Lautour-Mézery, etc. et leurs écrits, elle conclut dans la première partie que «le Traité de la vie élégante est un ouvrage qui a été fait presque en commun par Balzac et par les autres jeunes gens de la Mode» et que «même si le Traité de la vie élégante est composé d'éléments empruntés et s'il obéit aux critères commerciaux de la revue, cela ne nous empêche pas de voir l'originalité de Balzac et le côté sérieux de cet article» ${ }^{8}$.

Dans la deuxième partie, l'auteur analyse les personnages balzaciens qui passaient pour des dandys. Nishikawa met en relief les dandys tels que Maxime de Trailles, Eugène de Rastignac, Charles Grandet, Don Juan Belbidéro, Henri de Marsay, en se référant à ce que Balzac dit dans le Traité et elle classifie ces divers dandys et leurs différents caractères dans la Comédie bumaine. Nishikawa dit à la fin de son étude: «Dans les personnages de Dandys, Balzac matérialisa son désir et son rêve les plus intimes. Comme les dandys sur les grands boulevards avaient des foules à séduire, le romancier avait son public à attirer. (...) Balzac contribua à perpétuer le dandysme, qui en tant que mode, était destiné à une existence éphémère, et à le communiquer aux générations futures» ${ }^{9}$. Depuis 1990 à peu près, les ouvrages de Shigeru Kashima et de Toyoko Yamada qui décrivent la mode parisienne et le journalisme à Paris dans les années 1830 en tant que toile de fond de la civilisation et de la culture de l'époque gagnent beaucoup de lecteurs au Japon. Le livre de Nishikawa est digne du nom d'explorateur de cette lignée.

\section{Successeurs de Takayama}

En 1971, Takayasu Oya présente sa thèse de doctorat d'université Les Aspects du comique dans les Contes drôlatiques de Balzac à l'Université de Paris-Sorbonne. Sa thèse comporte deux parties: dans la première partie, Oya étudie ce qui fait le comique dans les Contes drôlatiques, et dans la deuxième partie, il analyse l'effet des techniques comiques tant au point de vue de la rhétorique que du vocabulaire. Sur le plan thématique, il relève trois éléments du comique: le ménage à trois, les circonstances bouffonnes et les thèmes sexuels, en nous donnant divers exemples tirés de ces Contes drôlatiques. Quant à l'effet comique, il explique en détail plusieurs techniques que Balzac emploie pour donner du brio à ses textes, tels que le style archaïque, le maniement habile des images (comparaison, métaphore et exagération), le raisonnement illogique, le truisme, l'interprétation arbitraire de faits historiques, l'étymologie burlesque, la parodie, la satire, les mots d'esprit, etc.

Comme on le sait, le texte des Contes drôlatiques est écrit dans un français pseudo-archaïque imité de Rabelais. Oya l'analyse soigneusement. il en tire adéquatement de bons exemples et fait valoir la technique de Balzac en tant qu'humoriste. Il dit en conclusion que «pour ne parler que des mots et des tournures, ils se sont présentés à l'esprit de Balzac en même temps; parfois plus tôt que les thèmes qui constitueront le noyau des contes et ils ne sont pas un simple moyen de communiquer une idée ou de lui donner une forme concrète; ils possèdent une force comique, même leur propre esthétique, susceptible par eux-mêmes d'inviter le lecteur au rire» ${ }^{10}$. L'étude de Oya

(8) Y. Nishkawa, Balzac et le Dandysme, p. 67, Midori no Yacata, Kyoto, 1977.
(9) Ibid., p. 172.

(10) T. Oya, Les aspect du comique dans les 
sur le comique dans les Contes drôlatiques constate et supplée Balzac et le comique dans «La Comédie humaine» de Maurice Ménard, «qui fait voir et goûter un Balzac drôle, décrivant dans sa drôle de Comédie toute la marche du monde» ${ }^{11}$.

La Signification du Cénacle dans La Comédie humaine de Osamu Nishio a été publiée en 1980 à Tokyo. Je me souviens très bien du jour où je l'ai trouvée dans la bibliothèque de la Maison de Balzac à Paris que je fréquentais à l'automne de l'année 1981 pour préparer ma thèse. J'ai été beaucoup encouragé et je me suis décidé à terminer mes études en lisant ce livre. Le travail de Nishio est une version remaniée de sa thèse de Doctorat de troisième cycle qui a été soutenue en décembre 1976 à l'Université de Provence. Sur la suggestion de son directeur de thèse, Jacques Viard, Nishio met d'abord en lumière les correspondances au point de vue de la pensée entre Balzac et Pierre Leroux. Ensuite, il se rapproche du problème du Cénacle, de son origine jusqu'à son rôle dans 1'histoire de la littérature française comme dans les romans de Balzac.

Le livre de Nishio comporte deux parties. La première partie, «La genèse du Cénacle», traite de la préhistoire du Cénacle, ceux de Sainte-Beuve, de Hugo, de George Sand, etc. et des rapports étroits entre ces cénacles et le milieu littéraire où se trouvent les personnages balzaciens, Louis Lambert, Horace Bianchon, entre autres. La deuxième partie, «La valeur du Cénacle», examine les sources et les résonances du Cénacle dans la réalité et dans la fiction et dans le deuxième chapitre de la deuxième partie, Nishio éclaircit la signification et le rôle du Cénacle à l'époque de la Comédie bumaine. En concluant son étude, il dit: «En fait, Balzac loin de concevoir le Cénacle comme un parti politique, l'avait chargé de représenter la «sainte solidarité», seule capable d'amorcer un nouvel avenir pour une société que la lutte des coteries vouait à la mort. Et c'est là, nous semble-t-il, une des principales significations du Cénacle» ${ }^{12}$.

Je n'oublierai jamais l'aimable sourire de feu Alfred-Gérard Nizet, lorsqu'il m'a proposé de publier ma thèse que je venais de soutenir à Paris en juin 1982. Je l'ai abrégée et remaniée tout de suite, et le livre est paru à la fm de l'année 1983. J'ai voulu dans cette étude montrer que les trois romans qui composent la trilogie des Célibataires, Pierrette, Le Curé de Tours et La Rabouilleuse, ne sont pas rassemblés d'une manière arbitraire par l'auteur. La distribution des personnages selon leur caractère minutieusement étudié, la ressemblance de leur ambiance scénique et des intrigues, entre autres, tout cela nous démontre que le rapport de ces trois romans reste très étroit. Concernant le compte rendu de mon travail, je me contenterai de citer ceux de Pierre Citron et de Mireille Bossis.

«Il (l'auteur) porte attention de façon nuancée et sensible non pas seulement à la lettre du texte, mais à son atmosphère et à ses implications. Les remarques qu'il fait par exemple à diverses reprises sur l'importance du feu et de l'eau en relation avec telle ou telle figure sont pleines de pénétration et d'intérêt. (...) Je ne lui reprocherais que de montrer une discrétion exagérée à l'égard de la sexualité, qui affleure si souvent chez les personnages de Balzac». (Pierre Citron ) ${ }^{13}$

«Takao Kashiwagi nous fait remarquer, à juste titre, que les célibataires ne sont jamais les bourreaux; ils sont trop bêtes pour ce rôle! Ils servent seulement de catalyseur toujours négatifs et finissent par être à leur tour, les victimes de héros aux dents

«Contes drôlatiques» de Balzac, thèse présentée en 1971 à l'université de Paris-Sorbonne, exemplaire dactylographié, pp. 129-130.

(11) P. BerthIER, «compte rendu» de Balzac et le comique dans AsLa Comédie bumaineAt de Maurice Ménard in L'année balzacienne, Paris, P.U.F., 1985 , p. 362.
(12) O. Nishio, La Signification du cénacle dans La Comédie humaine, France-Tosho, 1980, Tokyo, p. 174.

(13) P. Citron, compte rendu de La Trilogie des Célibataires d'Honoré de Balzac de Takao Kashiwagi in Lannée balzacienne, Paris, P.U.F., 1985, pp. 370-371. 
longues; mais comme catalyseurs, les célibataires sont stables et leurs sentiments, immuables dans leur stérilité. Un tel disfonctionnement psychique amène à se poser la question de son origine. Takao Kashiwagi n’y manque pas: «le père dénaturé et la mère effacée, voilà la structure fondamentale d'une famille de célibataire» nous ditil». (Mireille Bossis $)^{14}$

\section{La jeune génération en fleurs}

Hajimé Sawada prête attention à la dernière période de la production romanesque de Balzac: 1846-1848. Il a soutenu sa thèse Les Idées implicites de Balzac à partir de 1846 sous la direction de Pierre Citron à l'université de la Sorbonne Nouvelle-Paris III en 1986. Pourquoi cette période? Selon lui, c'est dans ces années que le romancier fait preuve d'un nouvel élan créateur. Les Comédiens sans le savoir, Le Député d'Arcis, Les Parents pauvres sont de cette époque, qui voit Balzac terminer également alors Splendeurs et misères des courtisanes.

L'étude de Sawada a pour objet de déceler les idées du romancier qui ne sont implicitement exprimées que dans ses derniers romans, pour bien décrire la vision du monde balzacien dans la dernière étape de sa transformation. Non seulement les personnages balzaciens reflètent les observations de Balzac sur la société et sur ses contemporains, mais ils portent aussi l'empreinte de ses idéaux et de ses rêves. Sawada analyse donc des personnages et des types selon quatre thèmes dominants dans les œuvres balzaciennes: la politique, la société, l'amour et l'amitié. A chaque thème est consacré un chapitre qui contient quatre sections: défmitions des idées maîtresses, caractéristiques des personnages avant 1846 et à partir de 1846 et enfm signification de l'évolution.

La recherche du mouvement interne des thèmes, tout en tenant compte de la vie de Balzac et du contexte historique, met en relief une vision profondément pessimiste. Elle finit par nous montrer l'essence du génie balzacien. Le romancier sait aussi mettre à profit même son pessimisme de plus en plus grandissant dans ses derniers romans où se rapprochent plus que jamais la réalité et la fiction. La thèse de Sawada, outre qu'elle éclaire la valeur fondamentalement critique de l'œuvre de Balzac en face d'un monde oppressif auquel il nous faut toujours résister, contribue au plaisir de découvrir certains mystères de la création romanesque dans La Comédie bumaine, surtout au niveau des personnages, qui sont les fruits de l'imagination de Balzac luimême ou de son observation du monde réel.

Si l'étude de Sawada traite de plusieurs chefs-d'œuvre de Balzac à une certaine époque, celle de Yoshié Oshita jette la lumière sur Balzac dramaturge. Sa thèse: Balzac et le théâtre a été soutenue en 1997 à Paris XII sous la direction d'André Lorent. Elle se compose de quatre parties. Dans la première partie, Oshita fait remarquer les liens entre l'œuvre romanesque de Balzac et le théâtre en étudiant le vocabulaire de La Cousine Bette et l'utilisation des techniques dramatiques dans Le Cousin Pons et La Rabouilleuse. Balzac insère, en fait, le vocabulaire théâtral suivant le déroulement d'un jeu de scène. Le romancier fait valoir également l'effet visuel et auditif pour décrire ses personnages. Les «gestes», les «regards» et plusieurs sortes de «sons» dans les romans sont minutieusement analysés.

(14) M. Bossis, «compte rendu» de La Trilogie des Célibataires d'Honoré de Balzac de Takao Kashiwagi, in «Studi Francesi», N. 89, 1986, pp. $325-326$.
Takao Kashiwagi a publié son deuxième livre en français sur Balzac: Balzac, romancier du regard, Paris, Nizet, 2002. 
La deuxième partie est consacrée essentiellement aux personnages «comédiens» et «comédiens sans le savoir», c'est-à-dire Madame de Langeais, la Cibot, Pons et Hulot. Après avoir montré le comédien dans ces personnages, Oshita met en relief Vautrin, le «criminel-farceur». Elle n'oublie pas les rapports entre le personnage de Vautrin et certains acteurs réels comme Frédérick Lemaître et leurs rôles. Elle constate que Robert Macaire et Georges de Germany, joués par Lemaître, exercent une influence considérable sur la création des personnages chez Balzac.

La troisième partie est l'étude de Balzac dramaturge. Cette partie est consacrée à l'analyse de la comédie Le Faiseur: les traits communs des personnages de Mercadet, Robert Macaire joué par F. Lemaître et Vautrin sont signalés en citant plusieurs preuves. Oshita ajoute, pour montrer la popularité du Faiseur, des tableaux des représentations à partir de 1851 jusqu'à 1996.

La dernière partie de sa thèse traite des différentes adaptations scéniques et filmiques de six romans balzaciens: La Duchesse de Langeais, La Rabouilleuse, Le Père Coriot, Le Cousin Pons, La Cousine Bette et Splendeurs et misères des courtisanes. Pour les décrire, Oshita déploie toutes ses connaissanœs profondes en la matière. Elle porte atttention, entre autres, à La Duchesse de Langeais adaptée par Jean Giraudoux, qui parvient à rétablir sur l'écran le monde romanesque de Balzac tout en puisant aux sources. La documentation des adaptations filmiques faites par Oshita est très précieuse. Elle l'établit depuis les années 1910 en leur donnant une appréciation.

En conclusion, elle dit que malgré le fait que Balzac soit considéré comme l'un des plus grands romanciers de tous les temps, il lui paraît indispensable de découvrir en lui un «homme de théâtre». Le romancier dévoile au lecteur, en tant que dramaturge du «théâtre du monde» (le mot est de Vautrin), l'organisation de la société d'alors en recourant souvent aux ressources terminologiques du théâtre.

A notre regret, les thèses d'Oya, Sawada et Oshita présentées brièvement ci-dessus, ne sont pas encore publiées ni en France ni au Japon. Espérons qu'elles le seront un jour ${ }^{15}$. Mais la thèse de Naohisa Uda, La Madone dans l'œuvre d'Honoré de Balzac, soutenue en 1996 à la Sorbonne-Nouvelle sous la direction de Philippe Berthier, a été publiée en 1998 chez Septentrion. Cette thèse, constituée de six chapitres, traite de «la position implicite mais prépondérante qu'occupent les avatars de la Madone dans l'œuvre de Balzac» ${ }^{16}$. Pour voir l'évolution de la figure mariale chez Balzac, Uda analyse l'œuvre balzacienne chronologiquement, depuis les romans de jeunesse jusqu'aux derniers romans.

Dans le premier chapitre, il remarque l'aspect de l'amour exprimé dans les œuvres écrites sous le pseudonyme de Horace de Saint-Aubin et la figure de Marie de Verneuil du Dernier Chouan qui devient le prototype de la Madone à partir de laquelle le romancier crée ses madones: l'amour et la mère. Le deuxième chapitre traite des œuvres de 1830 à 1832: Jésus-Christ en Flandre, La Peau de chagrin, L'Enfant maudit, Les Proscrits et La Femme de trente ans. Uda analyse les personnages de ces romans en détail en nous montrant les signes mariaux: la Vierge évoquée par Raphaël, la conception virginale chez Jeanne, Notre-Dame dans Les Proscrits. Les personnages eux-mêmes se superposent sur l'image mariale: Jeanne et Hélène (La Femme de trente ans), par exemple, sont analysées dans l'activité littéraire de Balzac. Le troisième chapitre est consacré à plusieurs romans de 1832 à 1834 où le romancier décrit principalement l'amour sacré. Uda examine les sentiments et les comportements des hérö̈nes, Mme

(15) Outre ces thèses, nous devons ajouter celle de Kazué Ornuré (1987), qui analyse minutieusement une nouvelle de Balzac. Elle a consacré ses efforts à éclaircir totalement une nouvelle balza- cienne assez briève: Gobseck. Je regrette de n'avoir pas eu le temps de la lire en détail.

(16) N. Uda, La Madone dans l'œuvre d'Honoré de Balzac, Septentrion, 1998, p.158. 
Wilemsens, Mme de Beauséant, Mme de Langeais, Eugénie Grandet, etc. Durant cette période, «tout mène à la sacralisation de l'amour et de la femme», dit $\mathrm{Uda}^{17}$.

Le chapitre quatre étudie en grande partie Le Lys dans la vallée et les Contes drôlatiques. Dans ces deux ouvrages préside «Madame la vierge». Mme de Morsauf, comme la belle Impéria, s'élève vers la sainteté par la subblimation, elle seule a la grâce d'obtenir le triple titre de Mère-Épouse-Reine. Après elle suivent des femmes qui n'abandonnent pas le spirituel dans l'amour dans un monde en déchéance. Ces hérö̈nes Vierges-Enfants sont traitées dans le chapitre suivant. Depuis 1840, Balzac raconte souvent la vie et le destin de jeunes filles pures: Pierrette, Rosalie de Watteville, Ursule Mirouët, Modeste Mignon, etc. Outres ces filles, Uda porte son attention sur deux dames exceptionnelles, Camille Maupin et Véronique Graslin, dont l'amour demeure à la fois pur et impur. A cette époque, la figure de la Mère-Épouse est en déclin dans le monde balzacien et la figure de la Fille-Vierge la remplàce.

Mais il ne faut pas oublier l'image de la Mère. Le dernier chapitre traite donc de la Vierge-Mère. Uda y étudie minutieusement le personnage d'Ágathe Bridau et de la Descoings dans La Rabouilleuse aussi bien que celui de Bette, et d'Asie dans La Cousine Bette. Elles sont les mères nourricières. Leur caractère est ambigu, le Bien et le Mal se mélangent en elles. Ainsi, la thèse de Uda parcourt presque tous les romans cardinaux de la Comédie humaine pour y poursuivre les traits figuratifs et sentimentaux de la Madone: Fille, Épouse, Mère, Médiatrice chez les héroïnes balzaciennes. Le travail de Uda est vraiment sérieux et approfondi.

Pour terminer la présentation des études balzaciennes par les chercheurs japonais en français, je dois ajouter Lettres de Mme Bernard-François Balzac à sa fille Laure Surville, présentées avec une introduction et des notes par Hachiro Kusakabé (Éditions Seizansha, Kyoto, 2000) et les travaux des jeunes balzaciens japonais qui sont en train de préparer leur thèse en France aussi bien qu'au Japon. Pour commémorer le bicentenaire de la naissance de Balzac, le groupe des études balzaciennes japonaises va publier un recueil d'articles en français. Le rapport de Takayasu Oya dans l'Année balzacienne donne et donnera désormais les informations sur les études publiées au Japon $^{18}$.

TAKAO KASHIWAGI

(17) ibid., p. 158.

(18) Il faut ajouter à la liste des balzaciens japonais qui a soutenu leur thèse en France le nom de Kyoko Murata. Sa thèse Les métamorphoses du pacte diabolique dans l'ouvre de Balzac soutenue à Paris VII en 2001 sous la direction de Nicole Mozet traite les métamorphoses du pacte (ou contrat) diablique dans les œuvres de Balzac en analysant les œuvres fantastiques comme Centenaire, Melmoth reconcilié, La Peau de chagrin aussi bien que les ouvrages réalistes, comme Le Père Goriot, Illusions perdues et Splendeurs et misères des courtisanes et La Cousine Bett. Elle a été publiée en 2003 par OMUP et Klincksieck. 